\title{
Simulation of Earthquake Response of High Building with Soil-structure System
}

\author{
ZHAO Yun ${ }^{1, a}$, WEI Hua ${ }^{1, b}$, WANG Haijun ${ }^{1, c}$ \\ ${ }^{1}$ College of Architectural Engineering, Shenyang University of Technology, Shenyang, Liaoning, \\ 110870, China
}

agreat20102001@yahoo.com.cn, ${ }^{\mathrm{b}}$ weiflower1973@yahoo.com.cn, ${ }^{\mathrm{c}} \mathrm{corresponding}$ author: wang_navy@hotmail.com

Keywords: simulation, high-rise structure, earthquake response, interaction of soil-structure

\begin{abstract}
The simulation technique(ST) is applied to simulating the earthquake response of a high building with a soil-structure system.In order to simulate the dynamic response accurately,the soil-structure interaction(SSI) is considered and a three-dimension spring is applied for the model.The time history curves of the displacement, velocity and velocity of the stucture are achieved. The results of analysis in this paper are very accord with engineering practices.
\end{abstract}

\section{Introduction}

More and more ngineering practices show that the SSI has a large effect on the earthquake damage of structures due to large deformation of the soil,and furthermore,the flexibility of the soil as a transmission medium of the seismic wave has a great influence on earthquake[1]. The dynamic response of building structure is connected with the dynamic characteristics of the foundation as well as the mass,stiffness and damping of the structure. For the reasons given above, the SSI effect can not be ignored when the earthquake response of stucture is simulated.

\section{Modeling of Structure}

An 18-story frame-tub structure, which is $54 \mathrm{~m}$ high, $44 \mathrm{~m}$ long and $16 \mathrm{~m}$ wide, is selected. Each layer is $3 \mathrm{~m}$ tall,the space between pillars is $12 \mathrm{~m}$ and the inner beams are uniform arrayed in the plane. The site soil belongs to hard clay. For computational convenience, the gravity density is valued at uniform $2700 \mathrm{~kg} / \mathrm{m}^{3}$ and the Poisson's ratio is equal to 0.2 . The frame in this paper is adopted for an interlaminar shear model and the mass of each layer is treated as a lumped mass with frame columns connecting the mass points. In this way, the structure can be modeled as a series multi-degree of freedom vibration system with lumped masses and its stiffness matrix is a band matrix.

The distributed models are adopted for elements[5].BEAM188 is utilized for the beam and pillar. SHELL63 is adopted for the roof and floor plate.Loads and boundary conditions are applied to the model.

\section{Analysis of Earthquake Response Considering Interaction of the Soil-structure System}

Analysis Model for the Soil-structure System. The mechanism of the SSI is complex.There are sevral kinds of models set up to simulate the SSI as follow:(1) the mass-spring-damping model which treats the foundation as a dynamic system composed of mass,spring and damping; (2) the half-space model counting the soil as a homogeneous semi-infinite elastic solid; (3)the coefficient of elasticity model based on the assumption of E. Winker and treating the interaction between soil and structure as a spring system;(4)the finite element model;(5) the coupling model with finite element, boundary element and infinite element [2,3].

The frame-tub structure is actually a space system whose earthquake response includes the horizontal translational motion, vertical vibration and torsion.In order to simulate the dynamic structure more accurately and reliably considering the SSI,in this paper, a three-dimension spring unit 
is adopted for the soil spring model.The three-dimension spring unit has two nodes and each has three degrees of freedom for translational motions as $u_{x}, u_{y}$ and $u_{z}$. One node of every spring unit is coupled with the node of the foundation element and the other is constrained in the $\mathrm{X}$ direction, $\mathrm{Y}$ direction and $\mathrm{Z}$ direction as shown in Fig.1.

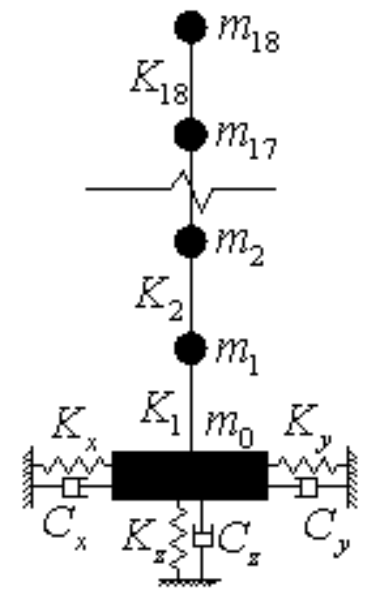

Fig.1. Schematic diagram of three-dimension spring

Parameters of Soil Spring Model and Equations of Motion. The effection by foundation on the structure can be equivalent to a spring coupled with the foundation boundary.The stiffness of the soil spring is expressed as $\overline{K_{g}}$ whose general form is as follow[2,3,4]:

$\overline{K_{g}}=i \omega C_{g}+K_{g}$.

Where $C_{g}$ and $K_{g}$ indicate the damping and stiffness matrix of the foundation respectively.

With the addition of a soil spring,the equation of free motion of the soil-structure system can still be expressed as follow:

$M \ddot{U}+C \dot{U}+K U=0$.

In Eq.2, the damping $C$ of the system is approximately equal to the damping $C_{g}$ of the foundation without regard to the distributed inherence damping of the structure.The stiffness matrix $K$ consists of the stiffness matrix $K_{s}$ of the structure and the stiffness matrix $K_{g}$ of the foundation while the mass matrix $M$ is counted as the mass matrix $M_{s}$ of the structure not considering the mass of the spring. From the above discussion, the equation of free motion of the soil-structure system can be expressed as follow:

$$
M_{s} \ddot{U}+C_{g} \dot{U}+\left(K_{s}+K_{g}\right) U=0 .
$$

However,the natural frequency of vibration of the soil-structure system is actually complex numbers due to inner damping of the structure,and Eq.3 can be expressed in another forma as follow[1,3]:

$$
\left[M_{s}(i \omega-\omega \xi)^{2}+C_{g}(i \omega-\omega \xi)+K_{s}+K_{g}\right](a+i b)=0 \text {. }
$$

Where $\omega$ and $\xi$ are respectively the frequency and damping ratio of the system,and $a$ and $b$ are the parameters about the displacement matrix.

By solving the equations above,we can obtain the natural frequency and mode of vibration of the system.

Earthquake Response of Structure with Soil Spring. The Newmark- $\beta$ method is applied to solution of the equation of forced motion of the structure as Eq.5:

$$
M \ddot{U}+C \dot{U}+K U=F \text {. }
$$

Where the parameters are similar to the ones in Eq.2 except $F$ which is the vector of external force dependent on time[3].

A correctional EI Centro(N-S) seismic wave with peak acceleration located at 70gal is acted on the structure model in the ANSYS Workbench.The natural frequencies of the first 5 modes of vibration are shown in Table1. 
Table 1. Natural frequencies of structure considering the SSI

\begin{tabular}{|c|c|c|c|c|c|}
\hline Mode of vibration & No.1 & No.2 & No.3 & No.4 & No.5 \\
\hline Natural frequency [Hz] & 0.34 & 0.67 & 0.98 & 1.02 & 1.21 \\
\hline
\end{tabular}

The time history curves of the 18th floor are listed as shown in Fig.2-Fig.5. For the convenience of comparison, the dynamic responses of the structure based on the rigid-foundation(labelled as $\mathrm{R}$ ) and of the structure with a soil-structure spring(labelled as S) are both shown in figures below.

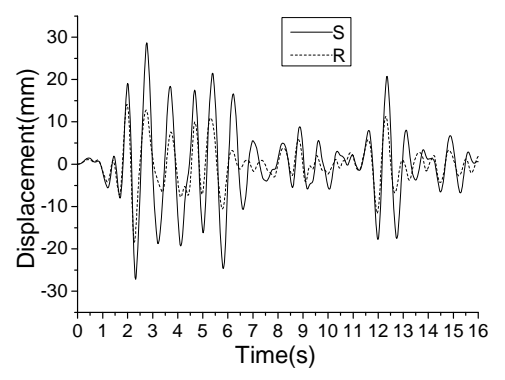

Fig.2. Displacement relative to ground

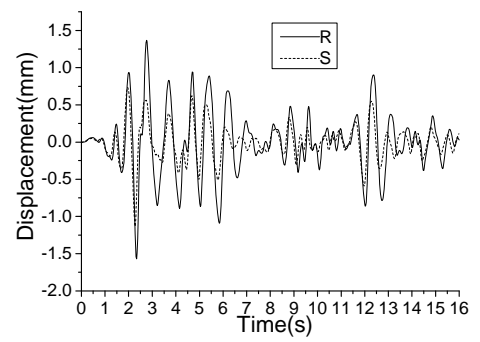

Fig.4. Relative storey displacement

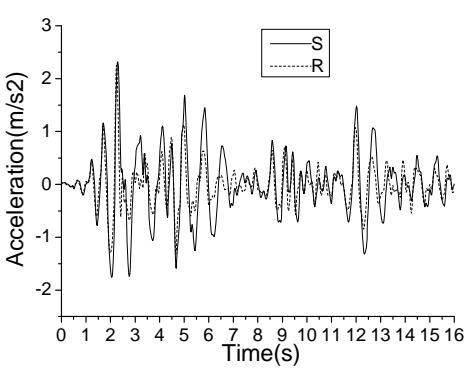

Fig.3. Absolute acceleration

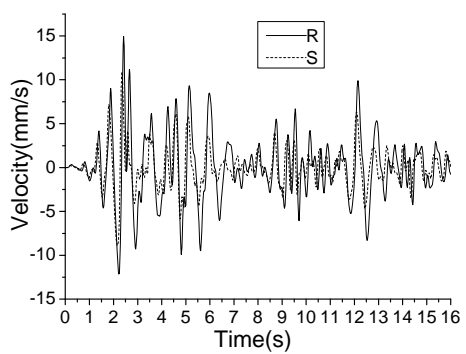

Fig.5. Relative storey velocity

According to the results of the simulation analysis, the first 5 modes of vibration considering the SSI basically reamin the same as those of the structure based on the rigid-foundation. It can be seen from Fig. 4 and Fig.5 that the displacement and the velocity between layers decrease by about $20 \%$ to $30 \%$,but the displacement relative to the ground and the absolute acceleration increase especially at the peak point of the curves as shown in Fig.2 and Fig.3.

\section{Summary}

The dynamic soil-structure interaction(SSI) can not be ignored.In this paper,the foundation has been treated as a mass-spring-damping system sufficiently considering the SSI and the results of the simulation analysis is consistent with the engineering practices. The concluding remarks can be summarized as follows:

1) Of the frequency spectrums of the foundation, the composition approximating the natural vibration frequency of the structure is intensified due to the SSI.

2) The flexibility of the foundation makes the basic cycle of the structure extend and the natural frequencie decrease.

3) The displacement and the velocity between layers of the structure decrease but the displacement relative to the ground and the absolute acceleration increase because of the SSI.Furthermore, the additional internal force caused by the $P-\Delta$ effect of the structure increases.

\section{Acknowledgement}

The work reported in this paper was supported by "Liaoning BaiQianWan Talents Program” Fund (2010921077), Liaoning Provincial Educational Department Fund (L2010412), Liaoning Provincial Educational Department Fund (L2010411), Shenyang Science and Technology Plan Fund (F10-205-1-08). The supports are gratefully acknowledged. 


\section{References}

[1] Y.C.Yang,D.L.Qian,C.Lei, Experimental Study on the Dynamic Interaction of Pile-soil-structure System by Shaking Table Test, Building Science Research of Sichuan, Vol.3 (2010): p.138-141

[2] J.B.Liu,Y.D.Lü,.A Direct Method for Analysis of Dynamic Soil-structure Interaction,China Civil Engineering Journal, Vol.31,No.3 (1998): p.55-64.

[3]Y.L.Jin,F.X.Wang,Nonlinear Seismic Response Analysis of Pile-soil-structure Interaxtion System, Coal Technology, Vol.26,No.6 (2007): p.1055-108

[4] X.L.Lü,P.Z.Li,B.Chen, Computer Simulation of the Dynamic Layered Soil-pile-structure Interaction System, Earthquake Engineering and Engineering Vibration, Vol.22,No.3 (2002):p. 91-99.

[5] W.Y.Wu,and S.G.Zhang, Research on Multimedia Simulation Environment for Wind-induced Tall Building, Engineering Mechanics, Vol.14,No.4 (1997): p.121-127. 